\title{
Luminosity function of contact binaries at high galactic latitudes towards the LMC and the SMC
}

\section{Journal Article}

\section{Author(s):}

Nef, Pascal D.; Rucinski, Slavek M.; Nef, Pascal D.; Rucinski, Slavek M.

Publication date:

2008

Permanent link:

https://doi.org/10.3929/ethz-b-000089864

Rights / license:

In Copyright - Non-Commercial Use Permitted

Originally published in:

Monthly Notices of the Royal Astronomical Society 385(4), https://doi.org/10.1111/j.1365-2966.2008.12994.x 


\title{
Luminosity function of contact binaries at high galactic latitudes towards the LMC and the SMC
}

\author{
Pascal D. Nef ${ }^{1 \star}$ and Slavek M. Rucinski ${ }^{2 \star}$ \\ ${ }^{1}$ Swiss Federal Institute of Technology (ETH-Zurich), 8093 Zurich, Switzerland \\ ${ }^{2}$ Department of Astronomy and Astrophysics, University of Toronto, 50 St George Str, Toronto, Ontario M5S 3H4, Canada
}

Accepted 2008 January 17. Received 2008 January 15; in original form 2007 October 4

\begin{abstract}
Using the Optical Gravitational Lensing Experiment (OGLE) catalogue of eclipsing binaries, 15 contact binaries were identified towards the Small Magellanic Cloud and the Large Magellanic Cloud at vertical distances from the Galactic plane between $300 \mathrm{pc}$ and $10 \mathrm{kpc}$. Based on the luminosity function calculated for these contact binaries, we estimated a frequency of occurrence relative to Main Sequence stars in the thick disc at roughly $\frac{1}{600}$. This estimate suffers from the small number statistics, but is consistent with the value previously found for the solar neighbourhood.
\end{abstract}

Key words: binaries: eclipsing - stars: luminosity function, mass function.

\section{INTRODUCTION}

During the past few decades, several attempts have been made to determine the local spatial density of contact binaries, with very diversified results. Rucinski (2002) (where full references are given and differences discussed) estimated their local spatial density at $(1.02 \pm 0.24) \times 10^{-5} \mathrm{pc}^{-3}$. Later, on the basis of the All Sky Automated Survey (ASAS), a relative frequency of occurrence (RFO) of one contact binary among about 500 solar-type stars was derived (Rucinski 2006), which is in full agreement with the mentioned spatial density. The RFO for contact binaries at high galactic latitudes, however, has been entirely unknown.

Here, we present an estimate for the spatial occurrence of contact binary systems relative to Main Sequence (MS) stars in the thick disc of our Galaxy, far from the Galactic plane. In particular, the luminosity function for contact binaries in two conical volumes towards the Small Magellanic Cloud (SMC) and the Large Magellanic Cloud (LMC) covering parts of the thick disc and the halo is determined. The results are then compared with the luminosity function for MS stars in the same region of the sky. The term 'contact binaries' is used here as a synonym for W UMa-type eclipsing binaries with orbital periods in a range of $0.22-1 \mathrm{~d}$. In this paper, for reasons explained below, we limit ourselves to a subset with periods $<0.45$ d.

\section{IDENTIFICATION OF SHORT PERIOD CONTACT BINARIES IN THE OGLE-CATALOGUE OF ECLIPSING BINARIES}

The Optical Gravitational Lensing Experiment (OGLE) was intended to detect dark matter in the Milky Way Galaxy using the microlensing technique, with the Magellanic Clouds and the

^E-mail: pnef@phys.ethz.ch; rucinski@ astro.utoronto.ca
Galactic bulge being the main targets of the survey (Udalski, Szymanski \& Kubiak 1997). As a byproduct, OGLE provides highquality, long-term photometry that can be used to analyse eclipsing binary stars. Two online catalogues (Wyrzykowski et al. 2003, 2004) were used for this study. They contain data in the standard photometric $B V I$ system for 2850 and 1351 eclipsing binaries, and cover an area of 4.6 and $2.4 \mathrm{deg}^{2}$ of the central parts of the LMC and the SMC, respectively. The OGLE-II survey has a faint limit of roughly $I=20.5 \mathrm{mag}$ with a corresponding error of $0.3 \mathrm{mag}$; the error becomes smaller for decreasing magnitude, reaching a bright limit of the survey at about 13 mag (Wyrzykowski et al. 2004).

The differentiation between contact binaries and other eclipsing binary types in the two OGLE catalogues was carried out by applying a contact binary criterion based on the Fourier analysis of the light curves, which was introduced by Rucinski (1997). ${ }^{1}$ By means of this criterion and using a visual inspection of the remaining light curves, we identified 10 and five contact binaries with orbital periods $P<0.45 \mathrm{~d}$ towards the LMC and the SMC, respectively. The light curves are plotted later in A1. Furthermore, we used the online data base from the Massive Compact Halo Object (MACHO) project (Alcock et al. 1997) to confirm the classification of the contact binaries in the OGLE catalogue. A direct comparison with the OGLE catalogue was only possible for the contact binaries towards the LMC because there is no MACHO photometry available for most survey fields covering the SMC. The MACHO photometry of the 10 contact binaries towards the LMC is in good agreement with the results obtained from the OGLE survey. ${ }^{2}$

\footnotetext{
${ }^{1}$ More detailed explanations for this and other techniques used in this paper can be found in Rucinski $(1997,2006)$.

${ }^{2}$ As an exception, the OGLE photometry in $V$ band for the contact binary OGLE050905.22-693315.1 was found to have a gross error of roughly 3 mag.
} 


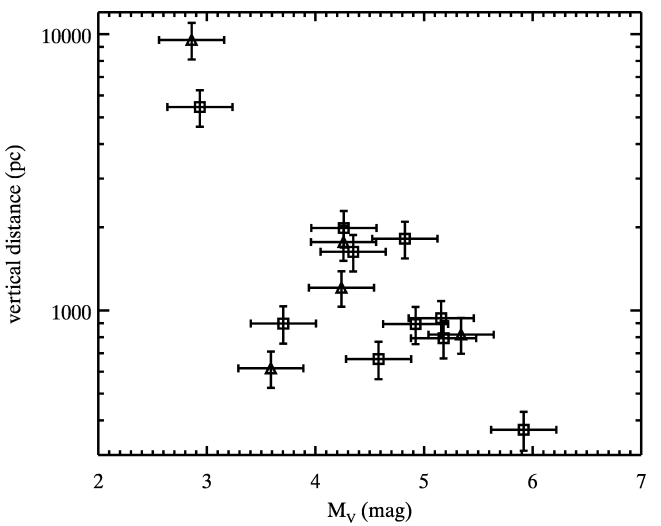

Figure 1. This plot shows the vertical distance from the galactic mid-plane for the 10 and five contact binaries towards the LMC (squares) and the SMC (triangles), respectively. The Sun is assumed to be located $30 \mathrm{pc}$ above the Galactic mid-plane.

The contact binaries in our sample have orbital periods in the range of $0.22-0.45 \mathrm{~d}$. The short period limit is a natural cut-off for contact binaries (Rucinski 2007), whereas the upper period limit was intentional: on one hand, we wanted to be sure of the contact binary classification and avoid semidetached binaries, while on the other hand, we wanted to use the simple $M_{V} \equiv M_{V}(\log P)$ calibration and avoid problems with uncertain or missing colour indices. Because the final RFO estimate is done using absolute magnitude bins of the luminosity function, the period limits signify an intentional restriction to the low-brightness end of the contact binary sequence. We will explain the details below.

\section{ABSOLUTE MAgNitudes AND DISTANCES}

Contact binaries show a correlation between the colour [i.e. $(B-$ $\left.V)_{0}\right]$ and the orbital period $P$, which was first observed by Eggen (1967). As was shown by Rucinski \& Duerbeck (1997), this correlation can be used to determine an absolute magnitude calibration $M_{V}=M_{V}\left[\log P,(B-V)_{0}\right]$; an even simpler version, $M_{V}=$ $M_{V}(\log P)$, is applicable (Rucinski 2006) when a restriction to short periods is added. In this research, the absolute magnitude calibration
$M_{V}=-1.5( \pm 0.8)-12.0( \pm 2.0) \log P$ was used, which is applicable for $P<0.562 \mathrm{~d}$ (Rucinski 2006). While applying this calibration to the contact binary sample, we arbitrarily assumed an uncertainty in absolute magnitude of $0.3 \mathrm{mag}$; the formal uncertainties in the calibration were larger and probably overestimated. Using this absolute magnitude calibration and the relation between the distance and the apparent and absolute magnitudes, $d=\operatorname{dex}\left(\frac{V-M_{V}+5-A_{V}}{5}\right)$, the distance from the Sun and the galactic height for the 15 contact binaries were estimated. The extinction towards the LMC and SMC was obtained from Schlegel, Finkbeiner \& Davis (1998); we assumed $A_{V}=3.1 E_{B-V}$. Fig. 1 illustrates the vertical distance from the Galactic mid-plane to the 15 contact binaries as a function of the absolute magnitude, whereas Table 1 lists the numerical results.

\section{THE AMPLITUDE DISTRIBUTION AND THE LUMINOSITY FUNCTION FOR THE 15 CONTACT BINARIES}

Small-amplitude systems often remain undetected in variable star searches when the photometric error is too large for detection of a small photometric variability. The number of missed small amplitude systems was estimated by comparing our sample with a theoretical model of the amplitude distribution for contact binaries (Rucinski 2001). This model predicts the amplitude distribution as a function of the degree of contact $f$ and the mass ratio of the two companions $q=\frac{M_{2}}{M_{1}}$ for a sample of contact binaries. In this research, we assumed $f=0.25$, what appears to be the favoured degree of contact, and a flat distribution of $q$. We applied this model in the same way as in Rucinski (2006), where more information can be found. Following this approach, we multiplied the number of contact binaries by factors of 1.6 and 1.5 for the LMC and the SMC, respectively; both factors have an uncertainty of about 20 per cent.

In order to determine the luminosity function for the 15 contact binaries, we simply used 1-mag wide absolute-magnitude bins by dividing the (corrected) number of stars in each bin by the respective volume $V=\frac{4 \pi}{3}\left(r_{2}^{3}-r_{1}^{3}\right) C . C$ denotes the fraction of the sphere covered by the survey and is equal to $1.09 \times 10^{-4}$ for the LMC and $5.82 \times 10^{-5}$ for the SMC. The results are given in Table 2.

Table 1. The short period contact binaries towards the LMC and the SMC. The table lists the name of the star (OGLE convention), the Magellanic Cloud field, the orbital period, the apparent magnitude in $V$ band, the absolute magnitude $M_{V}$, the distance along the line of sight and the vertical distance above the Galactic mid-plane.

\begin{tabular}{ccccccc}
\hline Name & Field & Period $(d)$ & $V(\mathrm{mag})$ & $M_{V}(\mathrm{mag})$ & Distance $(\mathrm{kpc})$ & Vertical distance $(\mathrm{kpc})$ \\
\hline OGLE003835.24-735413.2 (S1) & SMC & 0.26909 & 15.89 & 5.3 & $1.22 \pm 0.17$ & $0.82 \pm 0.12$ \\
OGLE005431.85-723510.9 (S2) & SMC & 0.33125 & 16.44 & 4.3 & $2.59 \pm 0.36$ & $1.77 \pm 0.26$ \\
OGLE005846.47-724315.3(S3) & SMC & 0.33244 & 15.61 & 4.2 & $1.78 \pm 0.25$ & $1.21 \pm 0.18$ \\
OGLE004619.65-725056.2 (S4) & SMC & 0.37663 & 13.55 & 3.6 & $0.93 \pm 0.13$ & $0.62 \pm 0.09$ \\
OGLE004849.85-725554.8(S5) & SMC & 0.43328 & 18.67 & 2.9 & $13.78 \pm 2.01$ & $9.54 \pm 1.44$ \\
OGLE054003.85-703837.3 (L1) & LMC & 0.24091 & 15.48 & 5.9 & $0.73 \pm 0.10$ & $0.37 \pm 0.06$ \\
OGLE050542.01-691725.9(L2) & LMC & 0.27755 & 16.31 & 5.2 & $1.51 \pm 0.21$ & $0.79 \pm 0.12$ \\
OGLE051932.28-694633.4(L3) & LMC & 0.27871 & 16.64 & 5.2 & $1.78 \pm 0.25$ & $0.94 \pm 0.14$ \\
OGLE053540.37-695413.9(L4) & LMC & 0.29159 & 16.30 & 4.9 & $1.69 \pm 0.23$ & $0.89 \pm 0.14$ \\
OGLE053251.73-700256.2(L5) & LMC & 0.29718 & 17.71 & 4.8 & $3.39 \pm 0.47$ & $1.82 \pm 0.28$ \\
OGLE053916.98-700903.3 (L6) & LMC & 0.31132 & 15.35 & 4.6 & $1.28 \pm 0.18$ & $0.67 \pm 0.10$ \\
OGLE050905.22-693315.1 (L7) & LMC & 0.32563 & 17.00 & 4.3 & $3.05 \pm 0.42$ & $1.63 \pm 0.25$ \\
OGLE053247.54-694403.1 (L8) & LMC & 0.33104 & 17.34 & 4.3 & $3.71 \pm 0.51$ & $1.99 \pm 0.30$ \\
OGLE053539.86-694759.5 (L9) & LMC & 0.36844 & 15.09 & 3.7 & $1.70 \pm 0.24$ & $0.90 \pm 0.14$ \\
OGLE054701.27-705623.3 (L10) & LMC & 0.42700 & 18.18 & 2.9 & $10.01 \pm 1.41$ & $5.44 \pm 0.82$ \\
\hline
\end{tabular}


Table 2. The luminosity function for the 15 close contact binaries towards the LMC and the SMC. The limiting distances are determined from $d=\operatorname{dex}$ $\left(\left(V-M_{V}+5-A_{V}\right) / 5\right)$ with apparent magnitude limits $V \in(13 \pm 0.3$, $21 \pm 0.3)$. The errors in the number of stars $n$ are calculated according to the Poisson statistics, which contribute to the uncertainty in $\phi$ as the main factor. Furthermore, we arbitrarily set the number of stars equal to one for the SMC bin centred at $M_{V}=6$ with zero star counts; an assumption which is consistent with the $1 \sigma$ Poisson error.

\begin{tabular}{lccccc}
\hline Cloud & $\begin{array}{c}M_{V} \\
(\mathrm{mag})\end{array}$ & $\begin{array}{c}r_{1} \\
(\mathrm{kpc})\end{array}$ & $\begin{array}{c}r_{2} \\
(\mathrm{kpc})\end{array}$ & $n$ & $\begin{array}{c}\phi \\
\left(\frac{\mathrm{stars}}{\mathrm{kpc}^{3}}\right)\end{array}$ \\
\hline SMC & 3 & 1.19 & 30.00 & 1.5 & $0.23 \pm 0.21$ \\
& 4 & 0.75 & 18.93 & 4.5 & $2.71 \pm 1.71$ \\
& 5 & 0.48 & 11.94 & 1.5 & $3.62 \pm 3.31$ \\
& 6 & 0.30 & 7.54 & 1.5 & $14.39 \pm 13.18$ \\
LMC & 3 & 1.13 & 28.41 & 1.6 & $0.15 \pm 0.14$ \\
& 4 & 0.71 & 17.93 & 4.8 & $1.82 \pm 1.12$ \\
& 5 & 0.45 & 11.31 & 8.0 & $12.10 \pm 6.59$ \\
& 6 & 0.28 & 7.14 & 1.6 & $9.63 \pm 8.60$ \\
\hline
\end{tabular}

\section{STELLAR DENSITY MODEL AND LUMINOSITY FUNCTION FOR MS STARS}

In order to establish the relative numbers of contact binaries, in relation to MS stars, we attempted to derive the predicted numbers of stars in the same search volumes as those of the OGLE and MACHO surveys. According to the pioneering work of Gilmore \& Reid (1983) and Bahcall \& Soneira (1984), the stellar distribution in the Milky Way can be modelled by a double exponential thin and thick disc and a spheroidal halo. Here, we describe the stellar density by such a three-component model

$n(z, R)=n_{\text {thin }}(z, R)+n_{\text {thick }}(z, R)+n_{\mathrm{sp}}(z, R)$,

where $(z, R)$ are the galacto-centric coordinates. The thick and the thin discs are modelled by

$$
\begin{aligned}
n_{\text {thin }}(z, R)= & n_{0}\left(1-c_{\text {thick }}-c_{\mathrm{sp}}\right) \\
& \times \exp \left[\frac{-|z|}{z_{\text {thin }}}\right] \exp \left[-\frac{R-R_{0}}{h_{\text {thin }}}\right] \\
n_{\text {thick }}(z, R)= & n_{0} c_{\text {thick }} \\
& \times \exp \left[\frac{-|z|}{z_{\text {thick }}}\right] \exp \left[-\frac{R-R_{0}}{h_{\text {thick }}}\right],
\end{aligned}
$$

where $z_{\text {thin }}$ and $z_{\text {thick }}$ denote the scaleheights and $h_{\text {thin }}$ and $h_{\text {thick }}$ the scalelengths for the thin and the thick disc, respectively. Furthermore, $n_{0}$ is the local stellar density, and $c_{\text {thick }}$ and $c_{\mathrm{sp}}$ are the localdensity normalizations of the thick disc and the halo relative to the thin disc. In this research, we use a projected de Vaucouleurs spheroid (Young 1976; Bahcall \& Soneira 1984) of the form

$n_{\mathrm{sp}}\left(R^{\prime}\right)=n_{0} c_{\mathrm{sp}}$

$$
\times \exp \left[-10.093\left(\frac{R^{\prime}}{R_{0}}\right)^{\frac{1}{4}}+10.093\right]\left(\frac{R^{\prime}}{R_{0}}\right)^{-\frac{7}{8}} .
$$

Here, $R^{\prime}$ is given by

$R^{\prime}=\left[R^{2}+\left(\frac{z}{\kappa}\right)^{2}\right]^{\frac{1}{2}}$

where $\kappa$ is the axis ratio of the de Vaucouleurs spheroid.

In our model, we used $z_{\text {thin }}=300 \pm 50 \mathrm{pc}, z_{\text {thick }}=1000 \pm 200 \mathrm{pc}$, $h_{\text {thin }}=2.5 \pm 1 \mathrm{kpc}, h_{\text {thick }}=3.5 \pm 1 \mathrm{kpc}, c_{\text {thick }}=0.05 \pm 0.03$, $c_{\mathrm{sp}}=0.0015 \pm 0.001, \kappa=0.55 \pm 0.1$ and $R_{0}=8 \pm 0.5 \mathrm{kpc}$. These parameters were chosen in consideration of the work of Gilmore \&

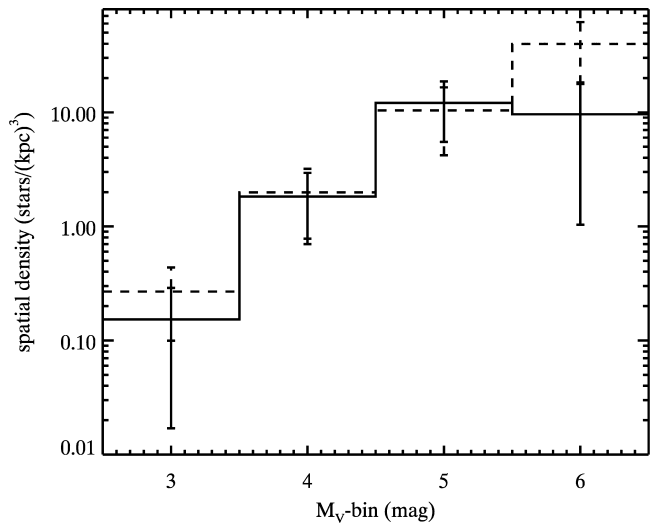

Figure 2. Luminosity function towards the LMC. The continuous line represents the contact binary luminosity function, whereas the dashed line represents the MS luminosity function divided by a factor of 650 .

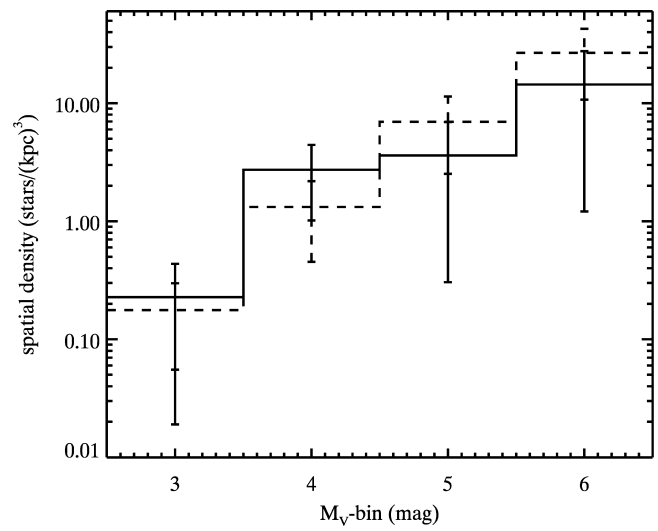

Figure 3. Luminosity function towards the SMC. The same as in Fig. 2 except for the scaling factor of 600 instead of 650 .

Reid (1983), Kuijken \& Gilmore (1989), Fux \& Martinet (1994), Bienaymé \& Séchaud (1997), Larsen \& Humphreys (2003) and Jurić et al. (2005). The rather large uncertainties in the parameters reflect ranges in the results obtained in the mentioned papers.

Wielen, Jahreiss \& Krüger (1983) derived a widely accepted luminosity function for MS stars in the solar neighbourhood using a sample of nearby stars, which we used to determine the stellar density in the solar neighbourhood $n_{0}$ as a function of absolute magnitude. With those results and the stellar distribution $n(z, R)$ as described above, we calculated the mean stellar density of MS stars in each volume corresponding to the four, 1-mag wide, absolute magnitude bins. By doing so, we were able to compare the derived contact binary luminosity function with the expected MS luminosity function for the same portion of the sky. The luminosity functions towards the LMC and the SMC are plotted in Figs 2 and 3, respectively. The contact binary luminosity functions were found to be best approximated by the MS luminosity function, if the latter was divided by 650 and 600 for the LMC and the SMC, respectively.

\section{CONCLUSIONS}

We conclude that each of the two samples yields the relative frequency of one contact binary among about $600 \mathrm{MS}$ stars in the two conical volumes towards the SMC and the LMC at galactic latitudes of $-44^{\circ}$ and $-33^{\circ}$, respectively. These estimates have uncertainties of roughly 50 per cent due to the large uncertainties arising from 
small number statistics and to the still uncertain parameters of the spheroidal component of the Galaxy. This component has been explicitly accounted for in the previous section and is estimated to contribute to the total number of stars in the SMC and the LMC search volumes at levels of about 30 and 20 per cent, respectively. The contribution varies with the $M_{V}$. Specifically, for the SMC, it changes from 38 per cent for the $M_{V}$ bin with the largest distances to 17 per cent for the $M_{V}$ bin centred at $6 \mathrm{mag}$; for the LMC, it changes from 29 to 10 per cent in the same range of $M_{V}$.

The results on the relative frequency of contact binary stars at large galacto-centric distances is consistent with estimates for the solar neighbourhood in previous work (Rucinski 2006). These are the very first data available on the contact binary distribution at high galactic latitudes.

\section{ACKNOWLEDGMENTS}

This work was done during the stay of PDN as an Exchange Undergraduate Student at the Department of Astronomy and Astrophysics of the University of Toronto. Thanks to the members of the department for hospitality and support. PDN would also like to thank Bryce Croll and Mirza Ahmic whose suggestions on how to improve the paper have been most helpful. Thanks are due to the OGLE and MACHO teams for making their results available for direct online access. Support from the Natural Sciences and Engineering Council of Canada to SMR is acknowledged with gratitude.

\section{REFERENCES}

Alcock C. et al. (The MACHO Collaboration), 1997, ApJ, 486, 697 Bahcall J. N., Soneira R. M., 1984, ApJS, 55, 67
Bienaymé O., Séchaud N., 1997, A\&A, 323, 781

Eggen O. J., 1967, Mem. RAS, 70, 111

Fux R., Martinet L., 1994, A\&A, 287, 21

Gilmore G., Reid N., 1983, MNRAS, 202, 1025

Jurić M. et al., 2005, preprint (astro-ph/0510520)

Kuijken K., Gilmore G., 1989, MNRAS, 239, 605

Larsen J. A., Humphreys R. M., 2003, AJ, 125, 1958

Rucinski S. M., 1993, PASP, 105, 1433

Rucinski S. M., 1997, AJ, 113, 407

Rucinski S. M., 2001, AJ, 122, 1007

Rucinski S. M., 2002, PASP, 114, 1124

Rucinski S. M., 2006, MNRAS, 368, 1319

Rucinski S. M., 2007, MNRAS, 382, 393

Rucinski S. M., Duerbeck H. W., 1997, PASP, 109, 1340

Schlegel D. J., Finkbeiner D. P., Davis M., 1998, AJ, 500, 525

Udalski A., Kubiak M., Szymański M., 1997, Acta Astron., 47, 319

Wielen R., Jahreiss H., Krüger R., 1983, in Philip A. G. D., Upgren A. R., eds, IAU Colloq. 76, The Nearby Stars and the Stellar Luminosity Function. L. Davis Press, Schenectady NY

Wyrzykowski L. et al., 2003, Acta Astron., 53, 1

Wyrzykowski L. et al., 2004, Acta Astron., 54, 1

Young P. J., 1976, AJ, 81, 807

\section{APPENDIX A: THE LIGHT CURVES OF THE 15 CONTACT BINARIES}

In Fig. A1 we show the light curves of the 15 contact binaries. The photometric data are taken from the two OGLE catalogues (Wyrzykowski et al. 2003, 2004). The fitted curves were obtained according to the approach of Rucinski (1993), i.e. using only the first five cosine and the first sine Fourier coefficients.

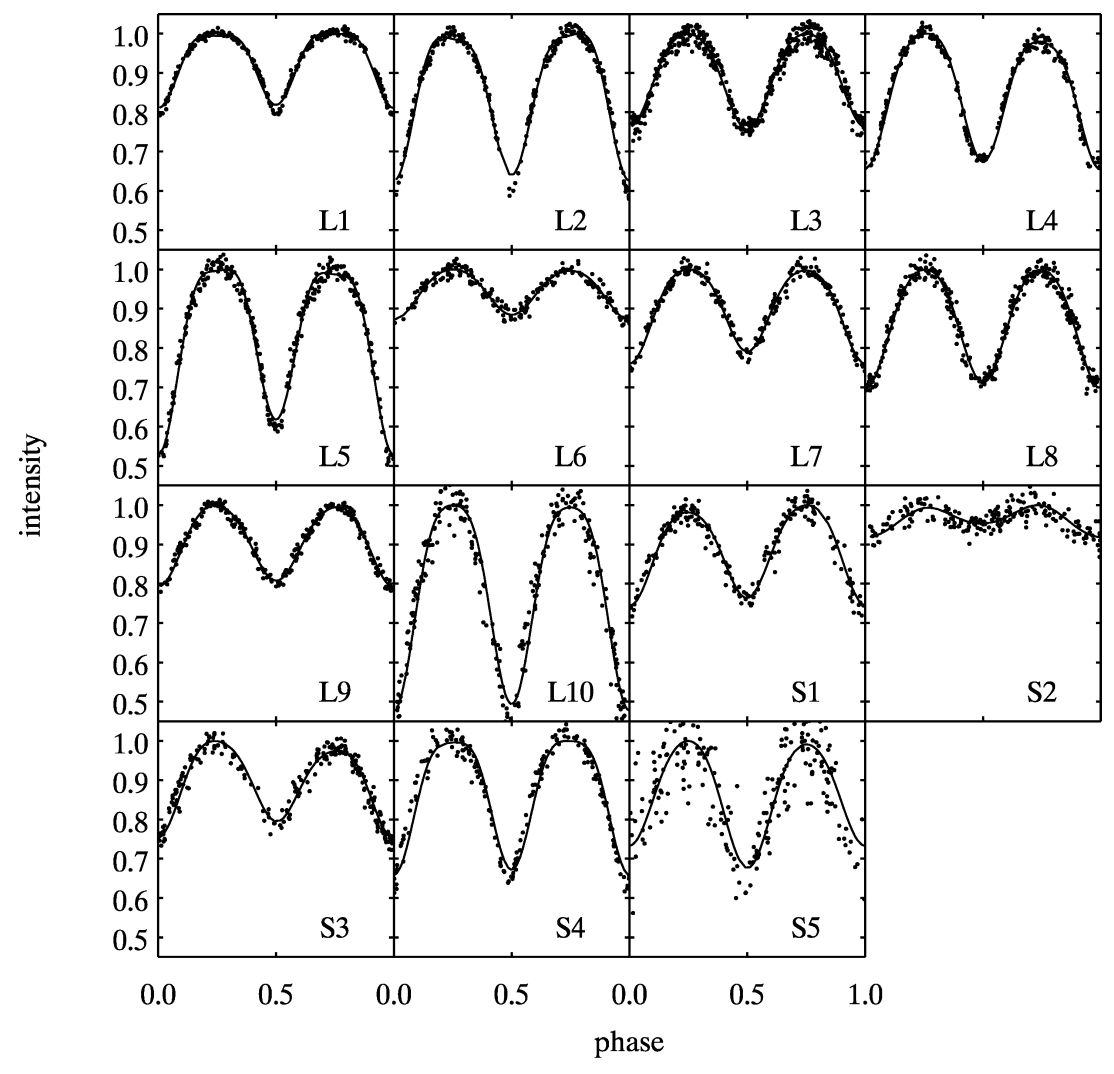

Figure A1. The light curves of the 15 close contact binaries. 